\title{
Experimental-Numerical Analysis of the Indentation-Based Damage Characterization Methodology
}

\author{
C.C. $\operatorname{Tasan}^{1,2}$, J.P.M. Hoefnagels ${ }^{2, \star}$, L.C.N. Louws ${ }^{2}$, M.G.D. Geers ${ }^{2}$ \\ ${ }^{1}$ Materials Innovation Institute (M2i), \\ P.O.Box 5008, 2600GA, Delft, the Netherlands \\ ${ }^{2}$ Eindhoven University of Technology, Department of Mechanical Engineering, P.O.Box 513, \\ $5600 \mathrm{MB}$, Eindhoven, The Netherlands \\ *e-mail: j.p.m.hoefnagels@tue.nl, tel:+31-40-2475894, Fax:+31-40-2447355
} Keywords: Sheet metal forming, ductile damage, microvoids, experimental-numerical
characterization

\begin{abstract}
The introduction of advanced high strength steels, e.g., into the automotive industry initiated a huge interest in analyzing and understanding ductile fracture of sheet metals to greater details. This demands for the development of experimental methodologies that provide microvoid evolution parameters, which also serve as crucial input parameters for advanced forming simulation that can predict damage evolution. Therefore, this work scrutinizes the reliability and applicability of an increasingly popular damage characterization methodology, in which microindentation tests are carried out to measure hardness and elastic modulus degradation as a function of accumulated strain, relating this degradation to damage evolution. To accomplish this goal, this methodology is applied to several different sheet metals of different formability (an interstitial-free steel, a dual phase steel, an aluminum-magnesium-silicon alloy and a ferritic stainless steel). To analyze and verify the results of indentation based methodology, damage evolution in these metals is monitored also via different experimental techniques, i.e. scanning electron microscopy, micro-ct tomography and sensitive density measurement. Moreover, finite element simulations are carried out to understand the effect of void accumulation in the degradation of hardness and elastic modulus. In the case of using the hardness as a damage probe, the degradation due to damage is always coupled to other effects (strain hardening, grain shape change, texture development) causing an increase in the obtained hardness value for all of the sheet metals tested, thereby complete obscuring any degradation of the hardness due to damage. In the case of elastic modulus, all the sheet metals tend to pile-up upon indentation when they are severely deformed, leading to large systematic errors in the Oliver-Pharr methodology based modulus determination, whereas the elastic modulus is also intrinsically altered by the grain shape change and texture development seen for increasing deformation. Therefore, it can only be concluded that, contrary to the published results in the literature, neither the hardness degradation nor the elastic modulus degradation can be used as a precise probe for damage accumulation, at least when the indentation based methodology is carried out in the originally-proposed manner that is commonly used in the literature.
\end{abstract}

\section{Introduction}

Last decades witnessed the introduction and increasing popularity of advanced high strength steels into the automotive industry. Despite their higher specific properties with respect to the conventional high strength low alloys steels, some grades of these alloys have been reported to suffer from unpredicted ductile failure, through damage evolution [1]. Since the predictive capabilities of available continuum damage models depend on the damage parameters provided by the experimental tools, there is a great interest nowadays to improve the experimental methods used to obtain these parameters. For example, a microindentation based methodology that uses hardness and/or elastic modulus degradation as a damage probe is one of the commonly used techniques to 
obtain damage evolution laws for a given material deformed in a prescribed strain path [2-5]. In this method, hardness/modulus of the metal in the 'damaged' situation is compared with those in the 'undamaged' situation to obtain a quantitative damage parameter at any given strain level. The local character of this methodology, together with its practical nature makes it a very promising method for industrial applications and scientific research.

However, published works in literature show some inconsistent results. Lemaitre et. al. (who were the first to propose this methodology) showed that the degradation of hardness could be used to determine the damage evolution in both low carbon steel (Figure 1a) and pure copper (Figure 1c) [2]. However, Cotterell et. al. (Figure 1d, [4]) for a similar low carbon steel and Guelorget et. al. (Figure 1b, [3]) for a similar copper alloy could observe no drop in the hardness as a function of strain (Figure 1b). Moreover, Alves reported that hardness based methodology gave good results with an aluminum alloy but not with a mild steel [5]. However, a (sudden) drop in hardness is crucial for the quantitative damage characterization methodology, i.e. a drop in hardness is needed to construct a fictitious undamaged hardness curve (which is effectively an extrapolation to larger strains of the hardness at lower strains before the drop in hardness [2]), which in turn is used to calculate the damage, D, at a certain strain level through:

$$
D=1-\frac{H}{H^{*}}
$$

where $\mathrm{H}$ is the measured hardness and $\mathrm{H}^{*}$ is undamaged hardness [2]. Similarly, the degradation of the elastic modulus due to damage can also used as a probe to study the damage evolution through:

$$
D=1-\frac{E}{E^{*}}
$$

where $\mathrm{H}$ is the measured elastic modulus and $\mathrm{H}^{*}$ is "undamaged" elastic modulus [2].

This present work aims to elucidate the reasons behind the inconsistency of data in the literature, in an effort to open new paths for precise indentation based damage quantification. This task is carried out via microindentation experiments on different materials of a wide scope of mechanical behavior and comparing the obtained results with the microstructural damage evolution as observed with alternative damage characterization methodologies including scanning electron microscopy, sensitive density measurements, and micro-ct tomography analysis. In addition, the microindentation results are compared with finite element simulations of the microindentation process of a damaged (voided) material.

\section{Experimental Methodology}

Experiments are carried out on interstitial-free steel (IF), X30Cr13 stainless steel (SS), dual phase 600 steel (DP) and aluminum 6016 alloy (Al) samples. Tensile samples from these sheets are tested (at $3 \mu / \mathrm{sec}$ ) under uniaxial loading using a micro-tensile stage (Kammrath\&Weiss GmbH), during which local strains are captured by digital image correlation (DIC) analysis, as shown in figure 2. 
(a)

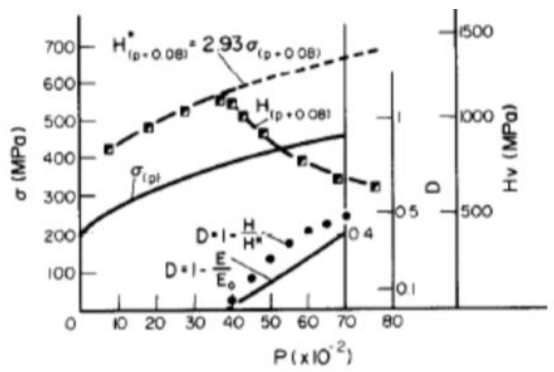

(c)

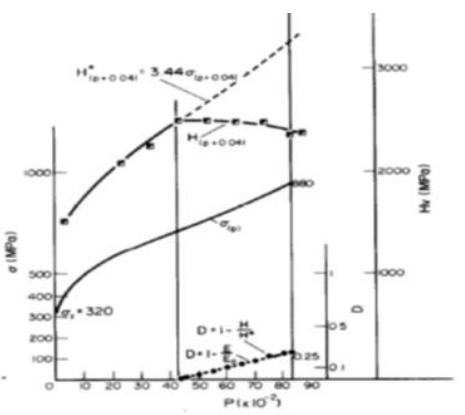

(b)

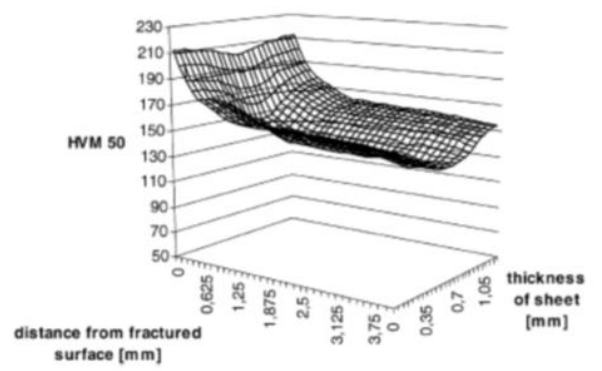

(d)

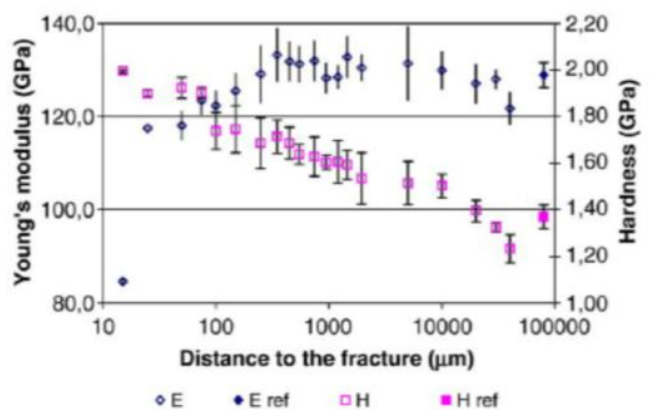

Fig. 1. Results from earlier works in which the microindentation based damage characterization methodology is used. For similar copper alloys, (a) Lemaitre et. al. observed a degradation of the hardness as a function of strain [2], whereas (b) Guelorget et. al. observed an increase of hardness, but reported a degradation of the elastic modulus [4]. [3]. And for similar low carbon steels, (c) Lemaitre et. al. reported a degradation of hardness [2], whereas (d) Cotterell et al. observed an increase in hardness

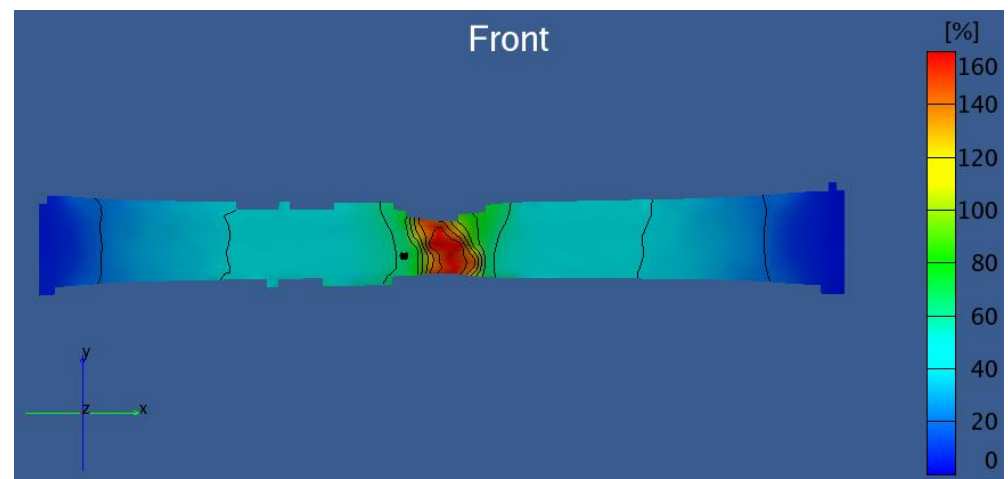

Fig. 2. Local von Mises strain in an interstitial-free steel uniaxial tensile specimen, showing a time frame just before fracture occurred. The color bar indicates the local strain in percentage. All DIC results have been obtained using the commercial 'Aramis'-software package from GOM GmbH.

All of the samples are tested in rolling direction. Cross sections of these specimens are polished (to a finish of $1 \mu$ roughness) to exclude the influence of roughness on the microindentation results. Displacement-controlled indentation tests are carried out with a Berkovich tip to a depth of 3500nm (figure 3(a)). To verify the reproducibility, indents are performed on both fractured parts of each specimen. The local hardness and elastic modulus of the tensile test specimens are obtained following the Oliver-Pharr methodology [6]. Hardness and modulus data is then coupled with the local strain data to investigate their evolution with increasing deformation. For direct evaluation of the damage accumulation, scanning electron microscopy, high-resolution micro-ct tomography (Nanotom ${ }^{\circledR}$; spatial resolution of $\sim 1 \mu \mathrm{m}$ ) and sensitive density measurements are carried out. For the scanning electron microscopy analysis, polished surfaces are slightly electro-polished to reveal the 
microvoids. For the micro-tomography and density measurements, small $(\sim 1 \mathrm{~mm} 3)$ cubes are cut out of tensile test specimen using electro-discharge machining (figure 3(b)).

(a)
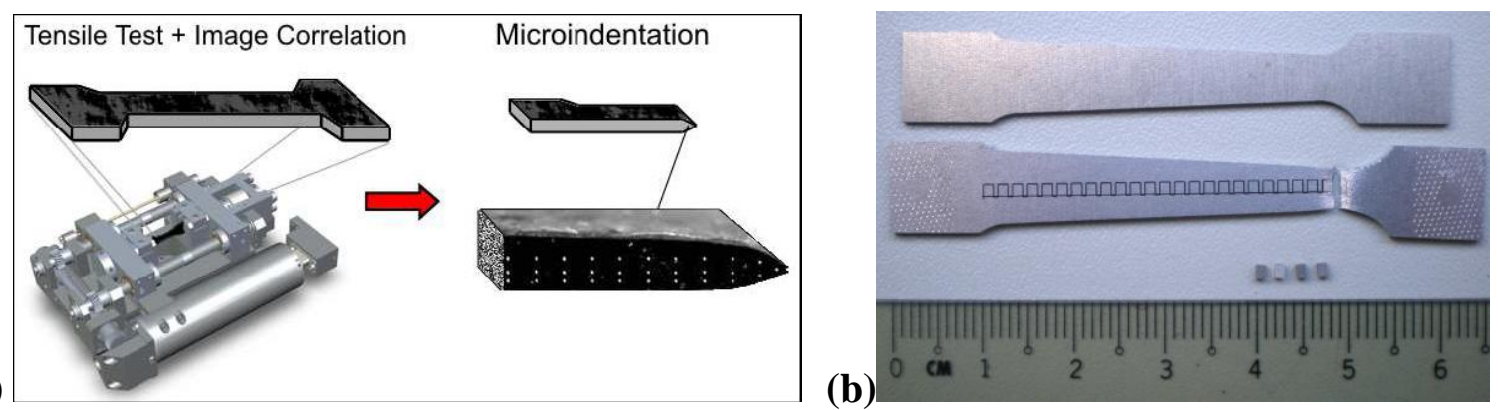

Fig. 3.(a) Tensile tests of sheet metal specimens are carried out with a tensile stage, after which microindentation tests are carried out on polished cross sections of the specimens. (b) Just below the cross-sectional surface using for indentation, $\sim 1 \mathrm{~mm} 3$ cubes are cut out to allow for microtomography and sensitive density measurements.

Finally, finite element simulations of microindentation tests of a damaged material are performed to find the trends for hardness and elastic modulus as a function of void fraction (figure 4). For these simulations, performed with MSC Marc Mentat (C), axi-symmetric boundary conditions with a surfacic void fraction are used, and the actual Berkovich tip used in the experiments is modeled as a rigid body. Voids are modeled explicitly with a random spatial distribution (taking into account a minimum distance between the voids) and a random void size distribution that is sampled from a void size distribution function as obtained from the SEM and micro-CT analysis. The material behavior is derived from tensile tests. Large number of simulations are done for each void fraction to finally get into averaged values for hardness and elastic modulus. Similar to the microindentation experiments, the simulations yield force-displacement indentation curves, from which the hardness and elastic modulus are obtained using the Oliver and Pharr methodology.

(a)

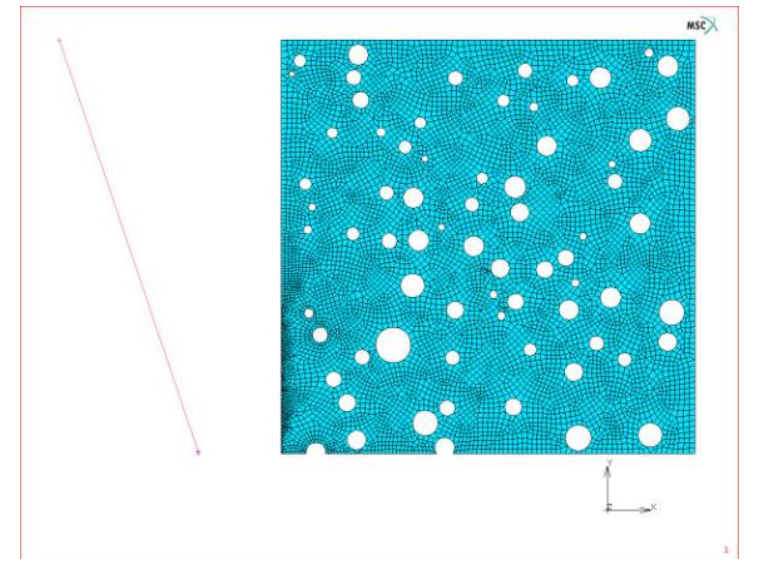

(b)

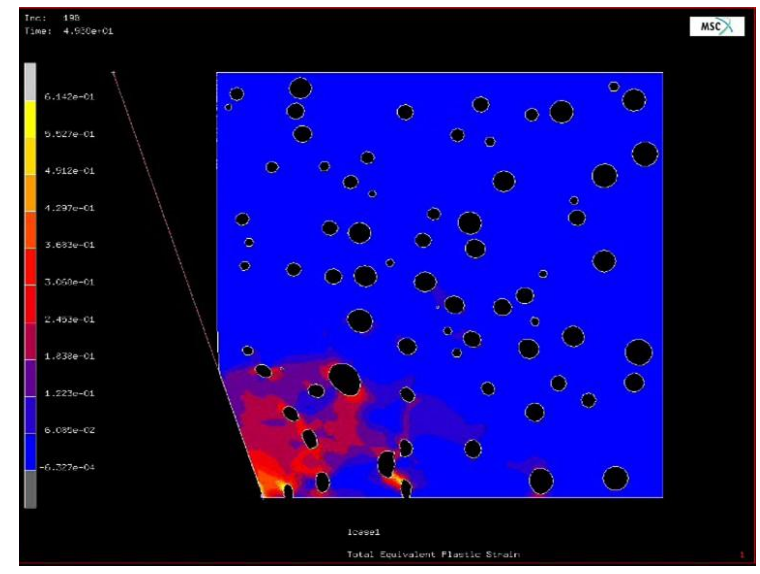

Fig. 4. (a) The MSC Marc Mentat (C FEM model used for verifying the indentation experiments, showing a typical void size distribution and spatial distribution as well as the automaticallygenerated FEM-mesh. (b) Typical indentation simulation, showing in color the von Mises strain distribution underneath the Berkovich indenter tip. 


\section{Results and Discussion}

Tensile curves of the four tested materials are given in Figure 5, where it is confirmed the four materials exhibit a wide range of mechanical behavior.

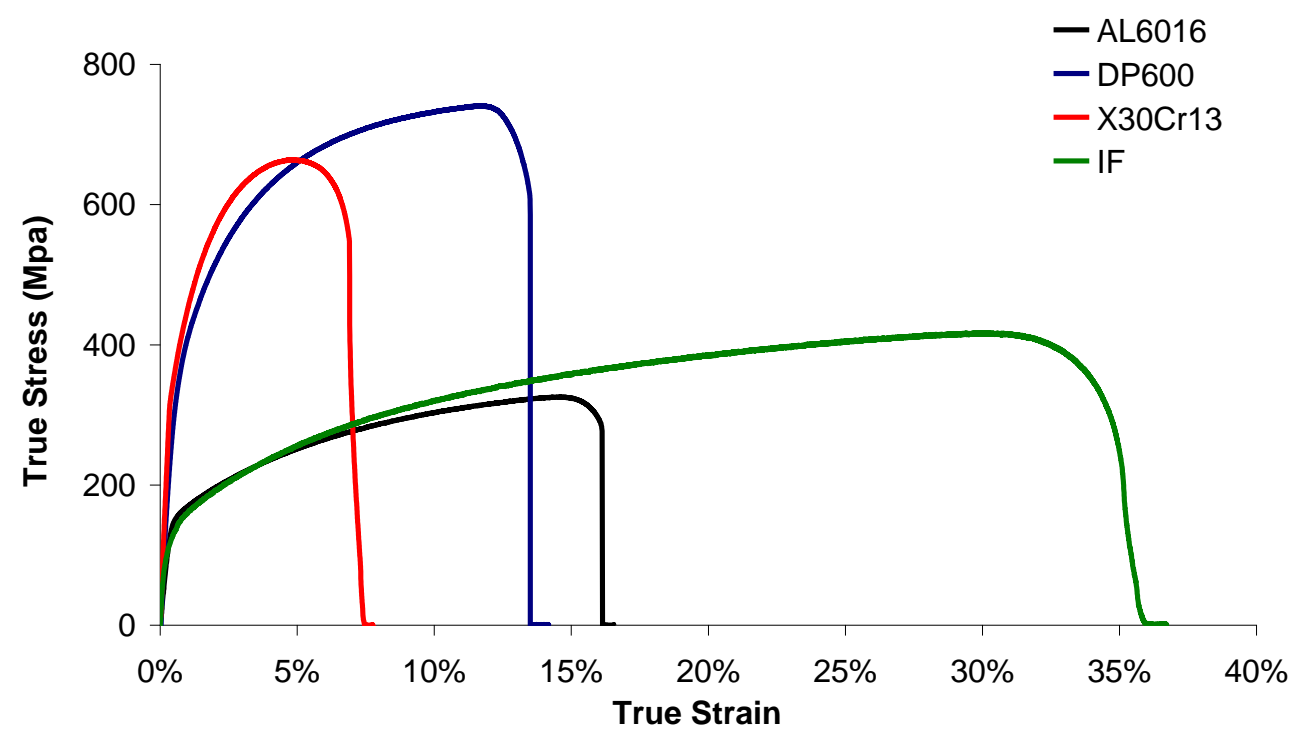

Fig. 5. True stress versus true strain of the four tested sheet metals, as measured under uniaxial tension.

Let us first focus on the hardness versus strain data, which shows a high degree of reproducibility, as shown in Figure 6. It is striking to observe that none of these metals of different formability show the decrease in hardness as observed by Lemaitre et. al [2]. Certainly no sudden drop is seen in the hardness which is needed to construct a fictitious undamaged hardness curve. At first sight this is surprising due to two reasons: First, the damage accumulation in these materials is clearly observed with other experimental tools: Figure 7 (a) shows a SEM image of the neck cross section of a DP600 specimen, showing extensive damage evolution in the regions where indentation tests are carried out. Similar electron microscopy results are obtained for the stainless steel and the interstitial-free steel. Furthermore, Figure 8 (b) shows one of the slices obtained with micro-ct tomography of the neck region of the A16016 specimen, where it is even possible to see large voids with a diameter of tens of micrometers located even hundreds of microns away from the neck region. This image also clearly show a strong inhomogeneity in the size and spatial distribution of the voids in the neck region of the aluminum specimen, however, a minimum void percentage of a few percents is seen throughout the neck region. Finally, sensitive density measurement - exhibiting a spatial resolution of somewhat than $1 \mathrm{~mm} 3$ - also revealed a significant drop in density towards the fracture surface ( 2.73423 to $2.71906 \mathrm{~kg} / \mathrm{l}$, leading to $0.55 \%$ damage). Second, numerical results also revealed a strong decrease of hardness with increasing void fraction (Figure 9 (a)), even at low void percentages, which is not unexpected as this dependence is the basis of the damage characterization methodology. 
(a)

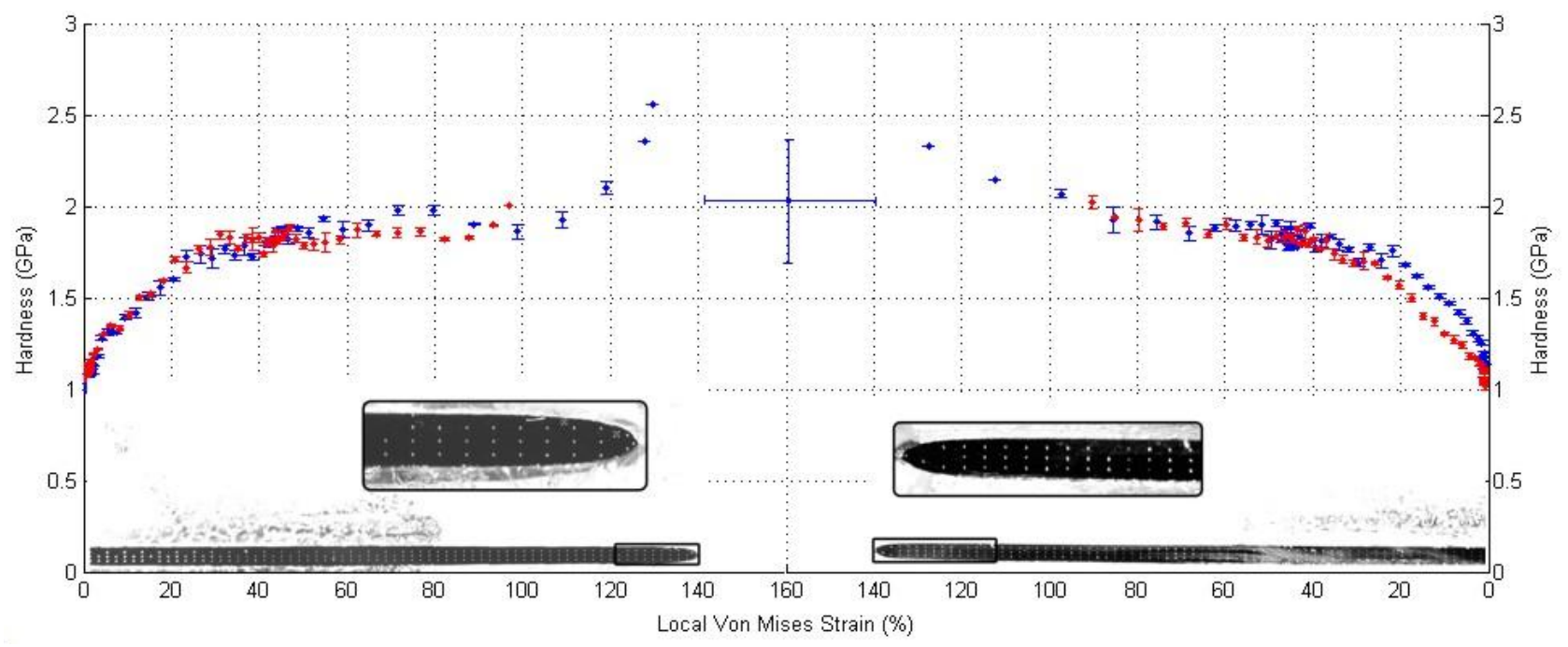

(b)

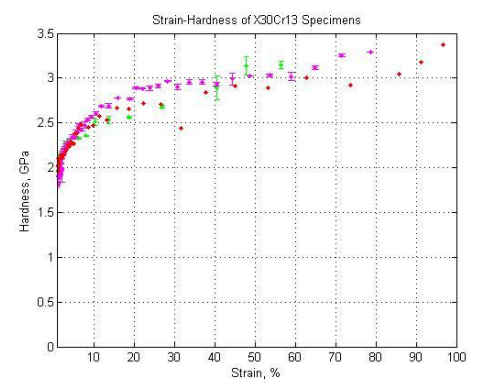

(c)

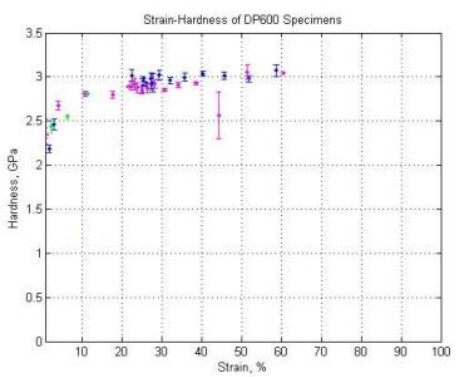

(d)

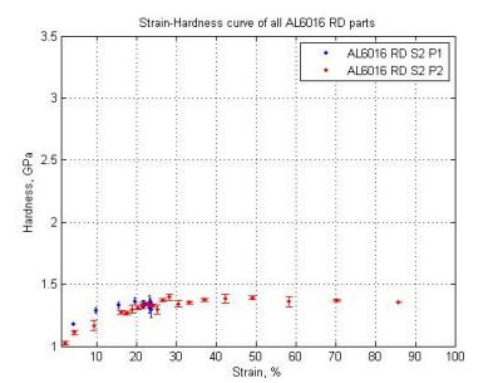

Fig. 6. Hardness versus local von Mises strain results of the sheets tested (a) IF steel, (b) X30Cr13, (c) DP600 and (d) Al6016.

To summarize, the existence of damage, i.e. microvoids, in the neck region is clearly confirmed and it is demonstrated that such a void density should cause a significant decrease in the hardness. Therefore, the inconsistence with the hardness measurements can only be explained when the decrease in hardness due to damage evolution is partially or fully obscured by other underlying factors arising due to the changes in the microstructure. The different possible factors that can cause an increase in hardness are best studied from interstitial-free steel, as it exhibits a late increase in hardness followed by what seem to be a decrease in hardness at ultimate strains (figure 6 (a)). First, a definitive factor involved is increase of hardness due to strain hardening, as it is generally known that for low amounts of damage accumulation strain hardening effects can obscure the damage effects in metals. For larger strains, the effect strain hardening may seem to decrease due to a decrease in strain hardening coefficient, however, because strain hardening is intrinsically coupled to damage resulting in the largest strains around the voids, its influence may well remain dominant even upto fracture. Another possible factor that can cause an increase in hardness is the significant change the shape of the grains for large deformation, i.e. the amount of grain boundaries underneath the tip is very different for different regions of a tensile test specimen: away from the neck, where the grains are still more-or-less equiaxed, the indenter tip 'encounters' much less grain boundary surface than at the neck, where the grains are severely deformed and elongated (Figure 7(b)). As interstitial free steel is the most deformable of the four metals testes, the grains near the fracture surface will be elongated the most, which explains why IF-steel shows the clear increase in hardness for large deformation (Figure 6 (a)). For the other metals, such a clear increase in hardness for large deformation is not seen, but that doesn't mean that the effect of grain shape change is not important for these metals. Finally, the strong elongation of the grains at large deformation must be accompanied by a strong change in texture, which may also have profound effect on the measured 
hardness. All these factors act together to yield a 'hardness' value at any given level of deformation. The effect of each is different in different microstructures (and therefore in different materials) which is the reason why this methodology, in its current configuration, is not applicable to most materials. Moreover, even in those cases where a significant drop in hardness is observed (e.g. figure 1(a) and 1(c)), one should be aware of the 'hidden' effects of strain hardening, grain shape change and texture development on the hardness values, which can easily cause a large systematic error in the values of damage parameter $\mathrm{D}$.

(a)

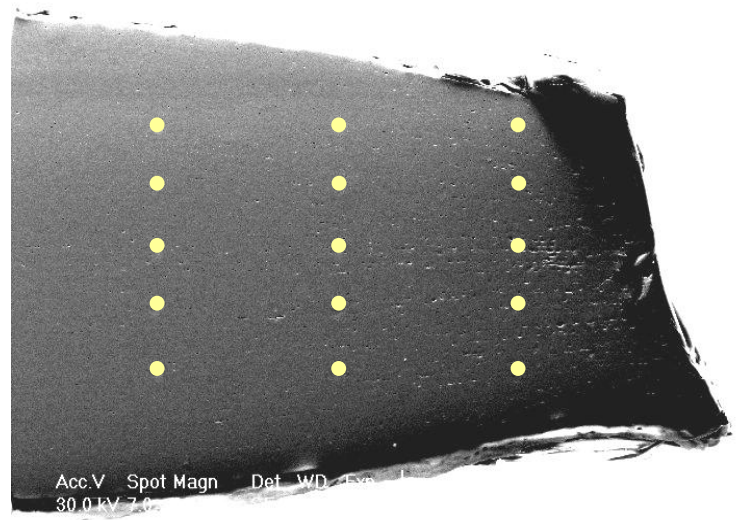

(b)

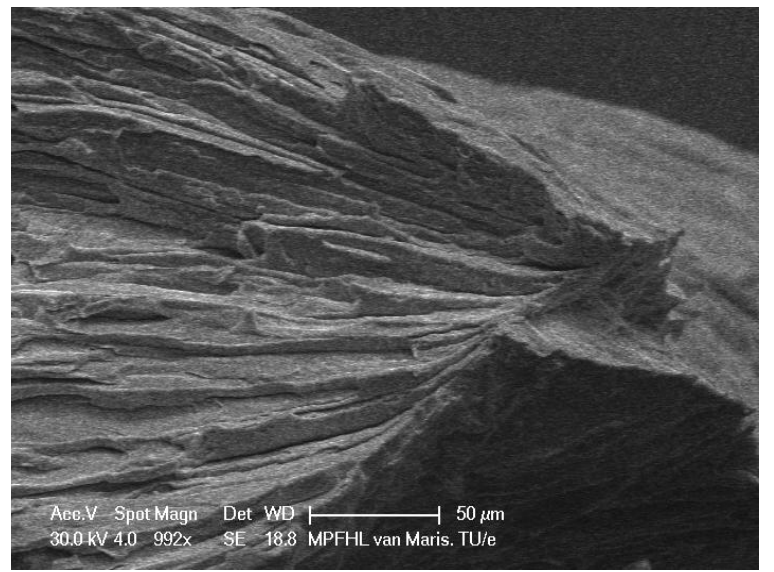

Fig. 7. Scanning electron microscopy images of the fracture region of a DP600 sample. (a) Microvoids and indent locations (yellow circles) as observed in the neck cross section. (b) Highly elongated grains in the localized neck of the interstitial-free sample.

(a)

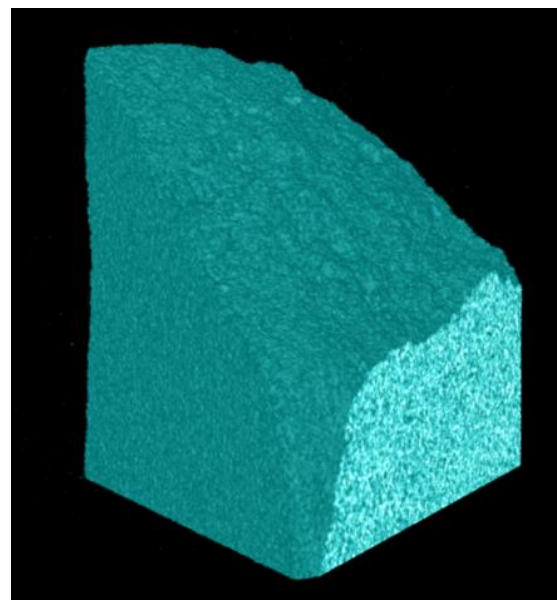

(b)

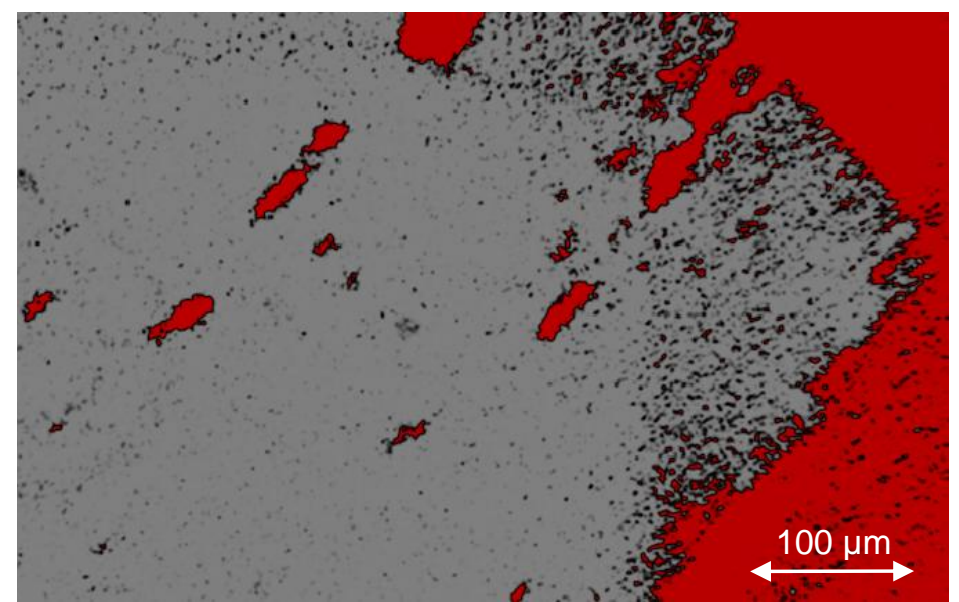

Fig. 8. (a) A 3D reconstructed micro tomography image of an EDM-ed test cube out of the neck of an aluminum tensile test sample, showing the morphology of the fracture surface. (b) Part of a slice through the fracture surface (on the upper right hand side of the image) showing the voids in the neck of the specimen. 
(a)

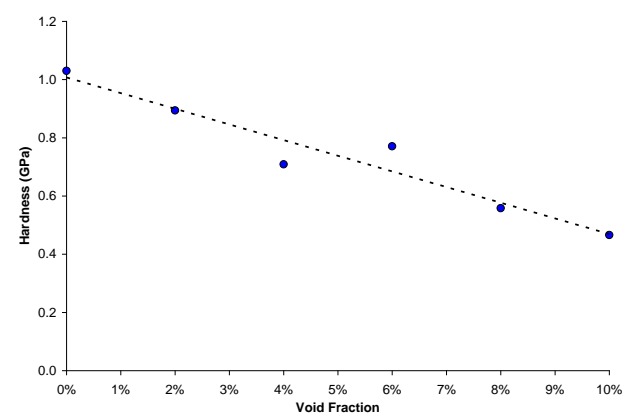

(b)

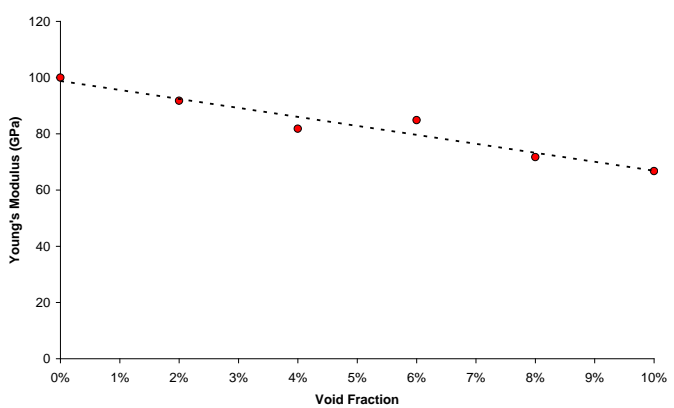

Fig. 9. Results of the microindentation simulations showing a decrease in both hardness (a) and elastic modulus (b) as a function of void fraction, even at very low void contents. The dashed lines are added as a guide to the eye.

(a)

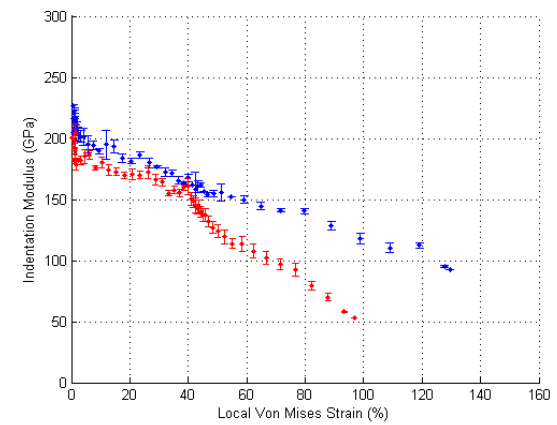

(c)

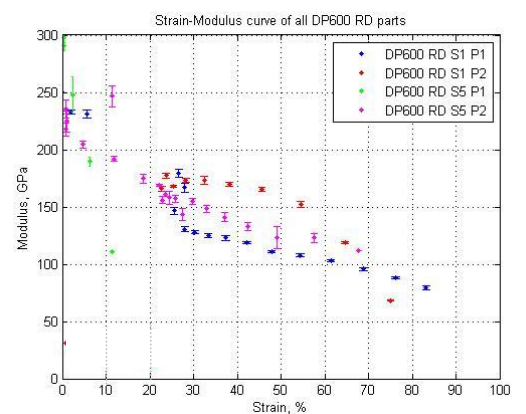

(b)

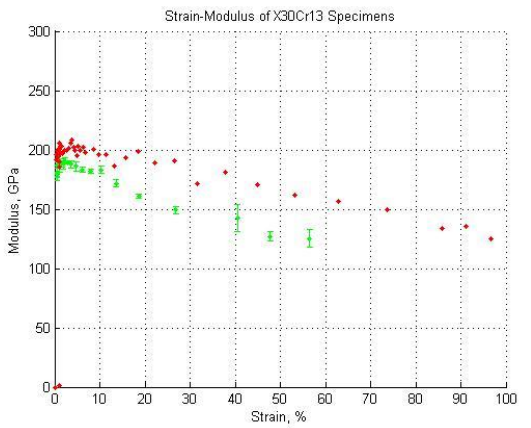

(d)

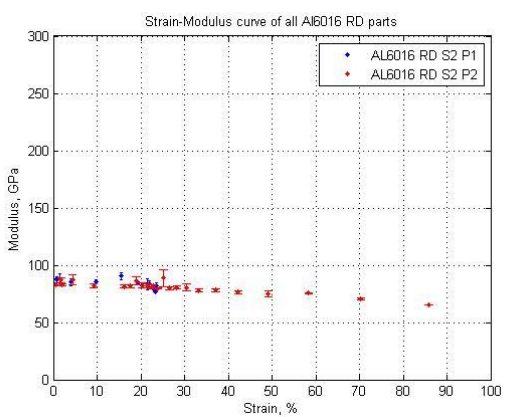

Fig. 10. Modulus vs. local von Mises strain results of the sheets tested (a) IF steel, (b) X30Cr13, (c) DP600 and (d) Al6016.

Let us now consider the coupled elastic modulus versus strain data presented in figures 10 . Even though the elastic modulus is determined from the same microindentation experiments as used to determine the hardness in Figure 6, it is clear that the statistical variation in the elastic modulus is much larger than that in the hardness. This large statistical variation is probably due to the fact that the unloading slope in the force-displacement curve, from which the elastic modulus is determined [6], is highly sensitive to variations in the local microstructure, which would explain why the statistical variation increases with increasing deformation. The large statistical variation already makes the elastic modulus an unpractical probe for damage quantification, however, for the present purpose it is more interesting to consider the systematic decrease in the elastic modulus with increasing strain and its correlation to damage evolution and other possible factors. Similar to the hardness curves, no sudden drop in elastic modulus, which may be attributed to a sudden onset of damage evolution, is observed. Apparently, if damage has a detrimental effect on the elastic modulus, then it must be a gradual effect. However, there are also other possible factors that can cause the observed decrease in elastic modulus. First of all, surface profilometry revealed for all four materials that indentation caused the material to sink-in when the material was undeformed or 
only lightly deformed before indentation, as is assumed in the Oliver and Pharr methodology. However, indentation caused the material to pile-up when the material was severely deformed prior to indentation, see Figure 10. In the Oliver and Pharr methodology, pile-up can cause a large underestimation of the elastic modulus due to a large underestimation of the contact area under the indenter tip [6]. In fact, pile-up also gives a systematic error in hardness, however, this effect is thought to be minor. Second, elongation of the grains as seen for large deformations (Figure 7(b)) can also cause a decrease in the elastic modulus, as elongated grains exhibit a larger ratio of grain boundary surface to internal grain volume, and the stiffness of a grain boundary is lower than that of the bulk crystal. Third, it is known that the elastic modulus also depends on texture, and especially for the strong texture that must be associated with the observed elongated grains this can be a significant effect. Finally, the elastic modulus also depends on residual stress, and for larger deformation a local residual stress is built in the material due to dislocation pile-up at the grain boundaries (strain hardening). In all, the observed decrease in elastic modulus can have many causes (some of which we might not have thought of yet), prohibiting its use as a quantitative probe of the accumulated damage.

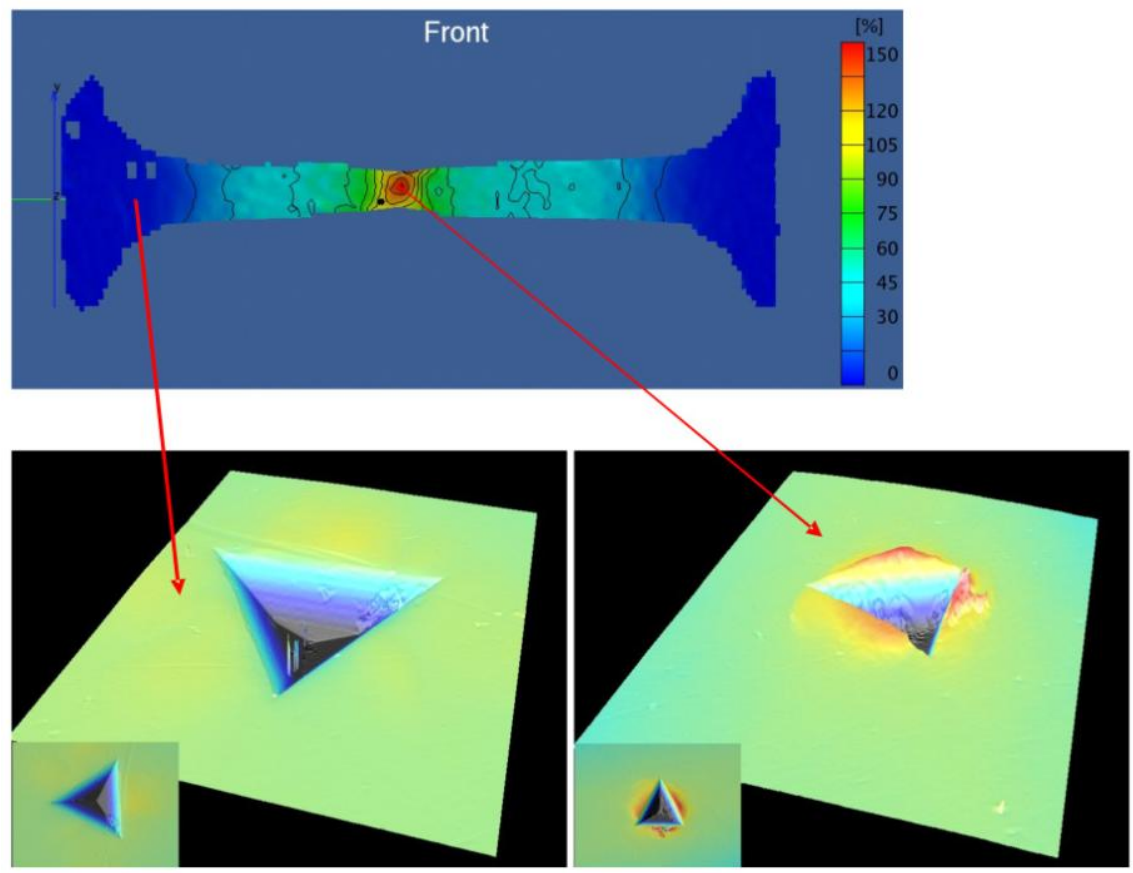

Fig. 11. Optical surface profilometry images of indents at two locations on the specimen: in the highly-deformed region near the crack and in a barely-deformed region far away from the crack.

\section{Conclusions}

This work critically analyzed the reliability and applicability of an increasingly popular damage characterization methodology, in which microindentation tests are carried out to measure hardness and elastic modulus degradation as a function of accumulated strain, relating this degradation to damage evolution. Four sheet metals of different formability were analyzed using a combination of microindentation, scanning electron microscopy, micro-ct tomography, sensitive density measurement, as well as finite element simulations, and the following consistent conclusions can be formulated: For increasing degree of deformation, both the harness and the elastic modulus not only change due to damage, but also due to 'hidden' factors, such as strain hardening, grain shape change, texture development, and indentation pile-up. This explains why for all four metals, the hardness keeps on increasing for increasing deformation or at least does not show a decrease, even 
though all three alternative diagnostics clearly show that there is a significant void density of at least a few percent at indentation locations and indentation simulations show that such a void density should definitively lead to a significant decrease in hardness in the case that the 'hidden' factors are not present. Only for the situation that a sudden drop in the hardness and the elastic modulus is observed, which can positively be attributed to damage using one or more alternative diagnostic, then either the hardness or the elastic modulus may be used as a probe for damage, however, still the 'hidden' factors will always modify its value in an unpredictable manner, making the hardness or the elastic modulus at best a qualitative probe for damage.

\section{Acknowledgments}

This research was carried out under the project number MC2.02114 in the framework of the Research Program of the Materials innovation institute M2i (www.m2i.nl), the former Netherlands Institute for Metals Research. Furthermore, the authors would like to thank Mark Heeren, Michiel Schellekens and Erica Coenen for their help with the results.

\section{References}

[1] S. Sadagopan, D. Urban, American Iron and Steel Institute / U.S. Department of Energy, Technology Roadmap Program, May 2003

[2] Lemaitre, J.; Dufailly, J., Engineering Fracture Mechanics, 28 (5/6), 643-661, 1987

[3] Cotterell M., Schambergerova J., Ziegelhamheim J. and Janovec J., Journal of Materials Processing Technology, 124, 293-296, 2002

[4] Guelorget B., François M. and Lu J., Materials Letters, 61, 34-36, 2007

[5] Alves M., Mechanics Based Design of Structures and Machines, 29(4), 451-476, 2001

[6] Oliver W.C. and Pharr G.M., Journal of Materials Research, 7 (6), 1564-1583, 1992 